\title{
Descripción del crecimiento en ovinos criollos utilizando el modelo Brody
}

\section{Using the Brody model for describing creole sheep growth}

\author{
Descrição do crescimento em ovinos crioula mediante \\ o modelo de Brody
}

\author{
Oscar Vergara-Garay'; Emiromel Llorente-M² ${ }^{2}$ Leonardo Ramos-C ${ }^{2}$; Moris Bustamante-Yánez ${ }^{3 *}$; \\ Juan C. Simanca-Sotelo ${ }^{*}$ \\ 1 Zoot, PhD, Universidad de Córdoba, Facultad de Medicina Veterinaria y Zootecnia, Grupo de Investigación en Producción \\ Animal Tropical. \\ $2 \mathrm{MVZ},{ }^{3} \mathrm{MVZ}, \mathrm{MSc},{ }^{4} \mathrm{MVZ},(\mathrm{c}) \mathrm{MSc}$ \\ * Universidad de Córdoba, Facultad de Medicina Veterinaria y Zootecnia, Maestría en Ciencias Veterinarias del Trópico. \\ Email: overgara@correo.unicordoba.edu.co
}

\begin{abstract}
Resumen
El objetivo de este estudio fue determinar la curva de crecimiento en una población de ovinos criollos en condiciones de pastoreo extensivo, utilizando el modelo Brody. Se utilizaron 448 pesajes de 59 animales nacidos entre los meses de agosto y septiembre del año 2012, pertenecientes al aprisco Altamar S.A, en el municipio de Buenavista, Córdoba (Colombia). Los estimados del modelo se obtuvieron mediante el procedimiento NLIN de SAS, considerando los efectos fijo de número de partos de la madre y número de crías por parto. Se encontró que el estimado para $\beta_{0}$ fue de $58.47 \pm 7.8$, para $\beta 1$ de $0.98 \pm 0.03$ y para $\beta_{2}$ de $0.0054 \pm 0.003$. No se encontró efecto significativo ( $\left.P>0.05\right)$ del número de partos sobre $\beta_{0}, \beta_{1}, y$ $\beta_{2}$. Se encontró efecto significativo $(P \leq 0.05)$ del número de crías por parto sobre $\beta_{0}$ y $\beta$ 1. El porcentaje de madurez estimada a los cuatro y siete meses fue $46.6 \pm 4.6$ y $66.1 \pm 4.5 \%$, respectivamente. La edad al $75 \%$ de madurez fue de $274 \pm 84$ días y al $95 \%$ de madurez de $596 \pm 202$ días. Los resultados de este estudio permiten concluir que los valores estimados para porcentaje de madurez y edad a la madurez en la población ovina fueron bajos, por lo que se debería implementar un programa de selección y mejorar el manejo zootécnico de los animales, con el fin que estos logren una madurez en el menor tiempo posible.
\end{abstract}

Palabras clave: edad a la madurez, modelación, parámetros, pesos.

\begin{abstract}
This study was aimed at using the Brody model for determining the growth curve for a creole sheep population kept on extensive grazing. The data came from weighing 59 animals (448 readings) born between August and September 2012 which were kept on Altamar SA's sheep-farm in Buenavista in the Córdoba Department in Colombia. Statistical Analysis System's (SAS) univariate nonlinear (NLIN) regression mode was used for obtaining the estimates, considering the fixed effects of the mothers' amount of births and the amount of lambs per birth. $\beta 0$ was estimated as being $58.47 \pm 7.8, \beta 10.98 \pm 0.03$ and $\beta 2$ $0.0054 \pm 0.003$. No significant effect was found on $\beta 0, \beta 1$ and/or $\beta 2(p>0.05)$ regarding the amount of births. A significant
\end{abstract}


effect $(p \leq 0.05)$ on $\beta 0$ and $\beta 1$ was found regarding the amount of lambs per birth. Percentage maturity estimated at four months was $46.6 \pm 4.6$ and $66.1 \pm 4.5 \%$ at seven months. Estimated age at $75 \%$ maturity was $274 \pm 84$ days and $596 \pm 202$ days at $95 \%$ maturity. It can be concluded from this study's results that the estimated values for percentage maturity and age at maturity in the target sheep population were low; this means that a selection programme should be introduced and the animals' zootechnical management improved to ensure maturity being reached in the shortest time possible.

Key words: age at maturity, modelling, parameter, weight.

\begin{abstract}
Resumo
O objetivo deste estudo foi determinar a curva de crescimento de uma população de ovelhas crioulas em condições de pastagem extensiva, utilizando o modelo Brody. Foram utilizados 448 pesando dados de 59 animais nascidos entre os meses de agosto e setembro de 2012, pertencentes ao rebanho Altamar SA, na cidade de Buenavista, Córdoba (Colômbia). As estimativas do modelo foram obtidos usando o procedimento SAS NLIN, considerando os efeitos fixos de número de partos da mãe e número de cordeiros por parto. As estimativas foram $58.47 \pm 7.8$ para $\beta 0,0.98 \pm 0.03$ para $\beta 1$ e 0.0054 \pm 0.003 para $\beta 2$. Nenhum efeito significativo $(P>0.05)$ no número de partos sobre $\beta 0, \beta 1$ e $\beta 2$. Houve efeito significativo $(\mathrm{P} \leq 0.05)$ no número de cordeiros por parto em $\beta 0$ e $\beta 1$. A percentagem de maturidade estimada em quatro e sete meses, foi de $46.6 \pm 46$ e $66.1 \pm 4.5 \%$, respectivamente. Idade de $75 \%$ de maturidade foi de $274 \pm 84$ dias e em $95 \%$ o vencimento de $596 \pm 202$ dias. Os resultados deste estudo, portanto, levaram à conclusão de que os valores estimados para percentual de maturidade e idade de maturação em ovinos foram baixos, por isso deve implementar uma selecção programa e melhorar o tratamento zootécnico de animais, a fim de eles atingem a maturidade no menor tempo possível.
\end{abstract}

Palavras-chave: idade em maturidade, modelagem, parámetros, pesos.

\section{Introducción}

En Colombia la población ovina ha sido fluctuante en los últimos años y el consumo de la carne ovina, ha ganado terreno en el mercado interno, creciendo a una tasa promedio marginal del orden del $1,5 \%$, por lo que se hace necesario asumir estrategias de desarrollo ovinocultor para crecer y posicionarse significativamente en el contexto pecuario actual, bien sea nacional o internacional, dadas las oportunidades comerciales existente (Arévalo y Correa 2013).

Por otro lado, la producción ovina en Colombia se caracteriza por la utilización de razas introducidas, razas criollas y cruzamientos alternos con el fin de fortalecer el vigor híbrido para la producción de carne. Estos sistemas productivos son extensivos y semi-intensivos, con tecnología de baja a media y por lo general están en manos de pequeños productores, que tienen poco acceso a la tecnología, aunque estas explotaciones cumplen una función económica importante, siendo el eje central de la agricultura familiar (Acero-Plazas 2014) y además están contempladas como una apuesta productiva, capaz de contribuir con los objetivos de productividad y competitividad (Parra et al., 2014).

Al considerar que la ovinocultura en el mercado nacional y de exportación posee ventajas comparativas, para lograr un incremento de la competitividad del sector se requiere la incorporación del conocimiento como factor producción y generador de ventajas competitivas mediante sistemas de gestión de conocimiento que se adapten a las condiciones de la producción primaria de un país en desarrollo como Colombia (Ospina et al., 2011)

De acuerdo a lo anterior, el objetivo de este trabajo fue estimar la curva de crecimiento mediante el modelo Brody en una población de ovinos criollos del departamento de Córdoba, Colombia, con el fin de caracterizar el crecimiento de estos animales en condiciones de pastoreo.

\section{Materiales y métodos}

\section{Localización}

El estudio se realizó en el Aprisco Altamar S.A, localizado en el municipio de Buenavista, departamento de Córdoba (Colombia). La zona se encuentra clasificada como bosque seco tropical, ubicada a $08^{\circ} 13^{\prime} 31^{\prime \prime}$ latitud Norte, $75^{\circ} 29^{\prime} 11^{\prime \prime}$ longitud Oeste, altura de 66 msnm, temperatura promedio de $27.3^{\circ} \mathrm{C}$, humedad relativa de $80 \%$, precipitación anual de $2269 \mathrm{~mm}$, brillo solar 1788 h/años y evaporación 1375 mm/año.

\section{Manejo de los animales}

Se utilizaron 59 corderos nacidos entre los meses de agosto y septiembre del año 2012, los animales fueron manejados en pastoreo extensivo en praderas de Brachiaria humidicola, Botrochoa pertuza y Brachiaria mutica, con disponibilidad de agua y sal mineral a voluntad. 
Las crías y las madres permanecieron en corrales con techos y estibas de madera, desde el nacimiento hasta los 5 días de edad, con suministro de pasto picado, agua y sal mineral a voluntad. Después de este periodo, las crías y las madres salían a pastorear, a partir de las 8:00 a.m. hasta las 4:00 p.m.

\section{Descripción de la información}

Para la toma de datos en campo, los animales fueron pesados al nacer y después cada 30 días de edad. El destete se realizó a los 90 días. Los pesajes fueron registrados en planillas diseñadas para tal fin. Igualmente, en dichos registros se registraron las fechas de los pesajes y las edades de los animales, fecha de servicio y de parto, número de corderos nacidos por parto, número de corderos muertos en el periodo pre y postdestete, identificación del padre y de la madre.

\section{Análisis de datos}

Para la determinación de la curva de crecimiento se utilizó el modelo propuesto por Brody (1946), usando el procedimiento NLIN del programa estadístico SAS. El modelo que describe el crecimiento propuesto por Brody es el siguiente:

$$
y_{i}=\beta_{0}\left(1-\beta_{1 e}{ }^{-\beta_{2} t_{i}}\right)
$$

Dónde:

$y=$ peso observado a la edad $\mathrm{t}$ (días).

$\beta_{0}=$ peso asintótico.

$\beta_{1}=$ constante de integración.

$\beta 2=$ tasa de madurez.

Además, se calculó el porcentaje de madurez $(M t)$ a los 4 y 7 meses de edad mediante la siguiente ecuación:

$$
M t=\left(1-\beta_{1} e^{-\beta_{2} t}\right)
$$

Dónde:

Mt $=$ porcentaje de madurez observado a 4 y 7 meses.

$t=$ tiempo para alcanzar un porcentaje de madurez (4 y 7 meses).

También, se calculó la edad para alcanzar el 75 y 95\% de madurez (EM) mediante la siguiente ecuación:

$$
E M=(\log (\beta 0-(\beta 0 * M t \%))-\log (\beta 0 * \beta 1)) /(-\beta 2)
$$

Dónde:

$E M=$ edad al alcanzar el 75 y $95 \%$ de madurez

$\mathrm{Mt} \%=$ porcentaje de madurez esperado (75 y 95\%).

\section{Resultados}

El estimado para $\beta 0$ fue de $58.47 \pm 7.8$, para $\beta 1$ de 0.98 \pm 0.03 y para $\beta 2$ de $0.0054 \pm 0.003$. En la Tabla 1 se presentan los estimados para porcentaje de madurez a los 4 y 7 meses y las edades al 75 y $95 \%$ de madurez.

Tabla 1. Promedios y desviaciones estándar para porcentajes de madurez a los cuatro y siete meses de edad y edad al 75 y 95\% de madurez de la población ovina estudiada.

\begin{tabular}{|c|c|c|}
\hline Variable & Promedio & $\begin{array}{c}\text { Desviación } \\
\text { Estándar }\end{array}$ \\
\hline Mt4 (\%) & 46.5 & 4.6 \\
\hline Mt7 (\%) & 66.1 & 4.4 \\
\hline EM75 (Días) & 274 & 84 \\
\hline EM95 (Días) & 596 & 202 \\
\hline
\end{tabular}

Mt4 = porcentaje de madurez a los 4 meses; Mt7 = porcentaje de madurez a los 7 meses; EM75 = Edad al alcanzar el 75\% de madurez; EM95= Edad al alcanzar el 95\% de madurez

En la Figura 1 se observa la curva de crecimiento en los ovinos criollos de acuerdo al modelo Brody.

Respecto a los efectos del número de partos de la madre y número de crías por parto, no se encontró efecto significativo $(\mathrm{P}>0.05)$ del número de partos sobre $\beta_{0}, \beta_{1}$ y $\beta_{2}$; mientras que el número de crías por parto presentó efecto significativo $(\mathrm{P} \leq 0.05)$ para $\beta 0 \mathrm{y}$ $\beta_{1}$ (Tabla 2).

Para Mt4, no se encontró efecto significativo ( $\mathrm{P}>0.05)$ del número de partos de la madre y del número de crías por parto, mientras que para Mt7 ambos efectos fueron significativos $(\mathrm{P} \leq 0.05)$. Para EM75, el número

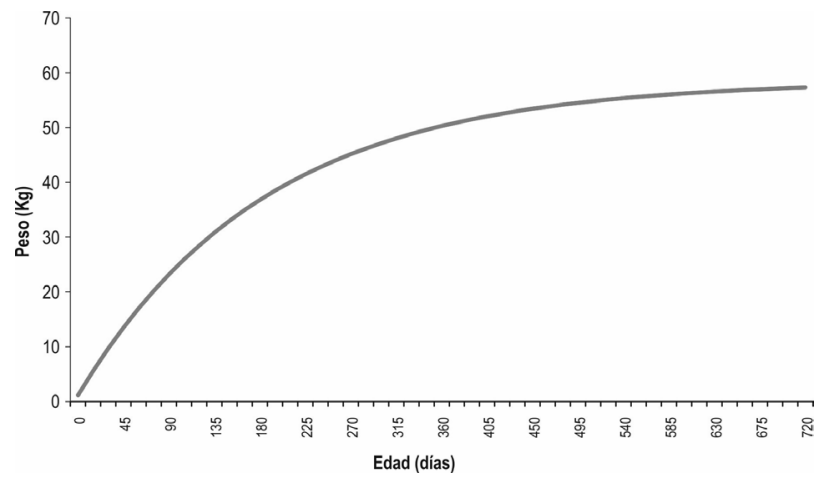

Figura 1. Curva de crecimiento de la población de ovinos de pelo estudiada de acuerdo al modelo Brody. 
de partos de la madre no fue significativo ( $P>0.05)$, pero si lo fue el número de crías por parto. Igual, sucedió para EM95 (Tabla 3).

Tabla 2. Promedios estimados para $\beta_{0}, \beta_{1}$ y $\beta_{2}$, según el número de partos de la madre y el número de crías por parto en la población ovina de pelo estudiada.

\begin{tabular}{|c|c|c|c|}
\hline $\begin{array}{c}\text { Número } \\
\text { de partos }\end{array}$ & $\beta \mathbf{0}$ & $\beta \mathbf{1}$ & $\beta 2$ \\
\hline 1 & $62.3^{\mathrm{a}}$ & $0.998^{\mathrm{a}}$ & $0.0047^{\mathrm{a}}$ \\
\hline 2 & $59.5^{\mathrm{a}}$ & $0.984^{\mathrm{a}}$ & $0.0051^{\mathrm{a}}$ \\
\hline 3 & $53.3^{\mathrm{a}}$ & $0.957^{\mathrm{a}}$ & $0.0062^{\mathrm{a}}$ \\
\hline 4 & $59.5^{\mathrm{a}}$ & $0.992^{\mathrm{a}}$ & $0.0055^{\mathrm{a}}$ \\
\hline 5 & $63.6^{\mathrm{a}}$ & $1.010^{\mathrm{a}}$ & $0.0045^{\mathrm{a}}$ \\
\hline 6 & $63.6^{\mathrm{a}}$ & $1.003^{\mathrm{a}}$ & $0.0044^{\mathrm{a}}$ \\
\hline \multicolumn{4}{|c|}{ Crías por parto } \\
\hline 1 & $56.7^{\mathrm{a}}$ & $0.975^{\mathrm{a}}$ & $0.0057^{\mathrm{a}}$ \\
\hline 2 & $63.9^{\mathrm{b}}$ & $1.006^{\mathrm{b}}$ & $0.0045^{\mathrm{a}}$ \\
\hline \multicolumn{4}{|c}{} \\
\hline
\end{tabular}

La Figura 2 muestra el comportamiento sigmoidal de la curva de crecimiento de ovinos criollos utilizando el modelo Brody, según el número de crías por parto.

La Figura 3 muestra el comportamiento sigmoidal de la curva de crecimiento en ovinos criollos utilizando el modelo Brody, de acuerdo al número de partos de la madre.

\section{Discusión}

El estimado de $\beta_{0}(58.47 \pm 7.8)$, indica el peso adulto de los corderos, es decir, representa la estimación del peso a la madurez o el valor límite del animal a una

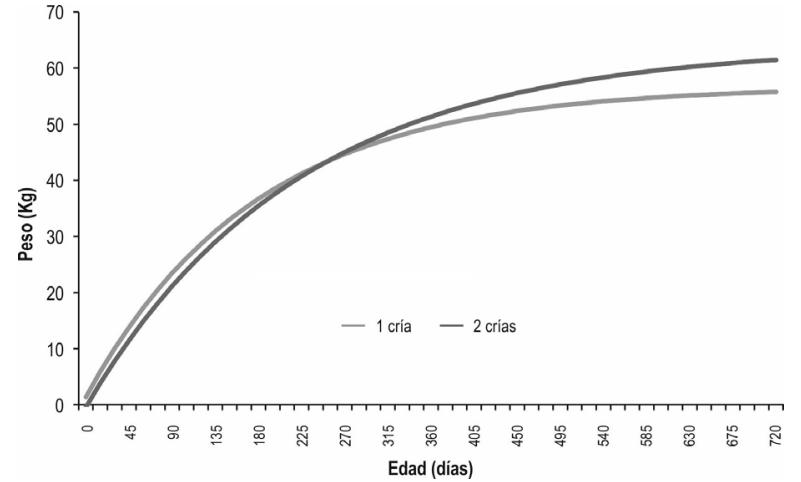

Figura 2. Curva de crecimiento de ovinos de pelo nacidos en partos simples y duplos de acuerdo al modelo Brody

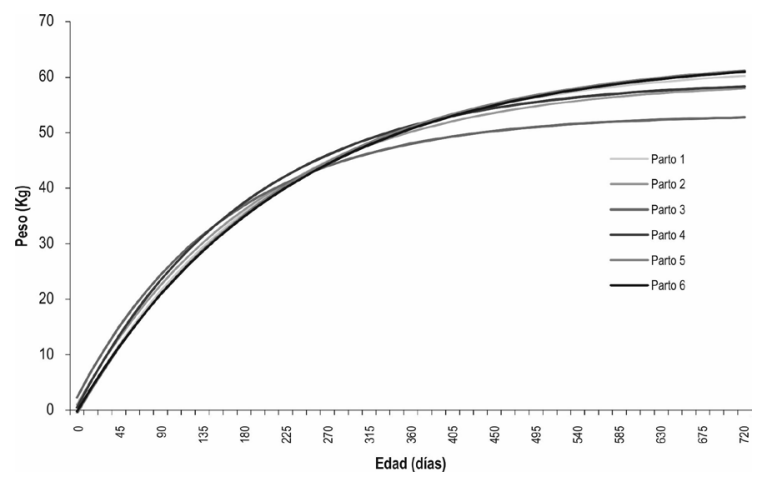

Figura 3. Curvas de crecimiento de ovinos de pelo según el número de partos de la madre de acuerdo al modelo Brody

edad adulta. Valores inferiores fueron reportados por Costa et al., (2014) y Bahreini et al., (2014), con estimados de 30.1 y 47.6, respectivamente. Igualmente, Souza et al., (2011) obtuvieron un peso asintótico de 28.1 en la raza Morada Nova, Santos (2012) en cruces de Santa Inés obtuvo un valor de 45.2 y Hossein-Zadeh y Golshani (2016) estimaron un valor de 29.1 en ovejas Guilen.

Tabla 3. Promedios estimados para porcentaje de madurez y edad a la madurez según el número de partos de la madre y el número de crías por parto en la población ovina de pelo estudiada.

\begin{tabular}{|c|c|c|c|c|}
\hline Número de partos & Mt4 (\%) & Mt7 (\%) & EM75 (días) & EM95 (días) \\
\hline 1 & $46.5^{\mathrm{a}}$ & $66.5^{\mathrm{a}}$ & $262^{\mathrm{a}}$ & $567^{\mathrm{a}}$ \\
\hline 2 & $45.9^{\mathrm{a}}$ & $65.3^{\mathrm{ab}}$ & $278^{\mathrm{a}}$ & $605^{\mathrm{a}}$ \\
\hline 3 & $46.9^{\mathrm{a}}$ & $65.8^{\mathrm{ab}}$ & $291^{\mathrm{a}}$ & $638^{\mathrm{a}}$ \\
\hline 4 & $47.7^{\mathrm{a}}$ & $67.6^{\mathrm{ac}}$ & $261^{\mathrm{a}}$ & $565^{\mathrm{a}}$ \\
\hline 5 & $46.5^{\mathrm{a}}$ & $66.6^{\mathrm{ab}}$ & $259^{\mathrm{a}}$ & $557^{\mathrm{a}}$ \\
\hline 6 & $46.0^{\mathrm{a}}$ & $66.1^{\mathrm{ab}}$ & $260^{\mathrm{a}}$ & $561^{\mathrm{a}}$ \\
\hline \multicolumn{5}{|c|}{ Crías por parto } \\
\hline 1 & $46.3^{\mathrm{a}}$ & $65.6^{\mathrm{a}}$ & $284^{\mathrm{a}}$ & $618^{\mathrm{a}}$ \\
\hline 2 & $46.9^{\mathrm{a}}$ & $67.0^{\mathrm{b}}$ & $253^{\mathrm{b}}$ & $546^{\mathrm{b}}$ \\
\hline
\end{tabular}


El valor obtenido para $\beta_{1}$ fue de $0.98 \pm 0.03$, siendo superior al encontrado por Santos (2012), Ullah et al., (2013) y Bahreini et al., (2014), quienes estimaron un valor de 0.92. Asimismo, Simanca et al., (2016), Teixeira et al., (2016) y Hossein-Zadeh y Golshani (2016) reportaron valores de 0.95 en ovinos F1 Santa Inés por Criollo, 0.92 en Santa Inés y 0.89 en Guilen, respectivamente.

El estimado de $\beta 2$ fue de $0.0054 \pm 0.003$, e indica la precocidad de madurez de los corderos. El valor estimado sugiere que los corderos criollos estudiados son poco precoces y la velocidad de crecimiento para llegar al peso asintótico fue lenta. Valores superiores fueron reportados por Malhado (2008), Souza et al., (2011), Ullah et al., (2013) y Bahreini et al., (2014), con estimados de 0.006, 0.006, 0.12 y 0.007, respectivamente. Por su parte, Santos (2012) y Simanca et al., (2016) reportaron valores inferiores (0.0041 y 0.0034$)$ al determinado en este estudio.

El porcentaje de madurez a los cuatro meses hallado fue de $46.5 \pm 4.6 \%$ y a los siete meses (Mt7) fue de 66.1 \pm 4.5. De estas dos edades de madurez, la más importante es Mt7, la cual es la edad tenida como referente en los rebaños de la región como la edad apropiada para llevar a sacrificio a los corderos y las hembras a la reproducción. Los valores de los porcentajes de madurez son bajos con relación a la edad, por lo tanto es pertinente mejorar algunas condiciones de alimentación y manejo en los sistemas productivos. Un porcentaje de madurez a los cuatro meses de edad similar al encontrado en este estudio fue reportado por Simanca et al., (2015) en ovinos de pelo $(45.6 \%)$ en el departamento de Córdoba

La edad para alcanzar $75 \%$ de la madurez fue de 274 \pm 84 días y para alcanzar el $95 \%$ de madurez fue de 596 días \pm 202 días, en consecuencia las edades en que los animales alcanzan la madurez son muy altas, lo que sugiere que la velocidad de crecimiento es lenta, por lo tanto es importante tomar medidas tendientes a aumentar los parámetros de en este rebaño. Entre estas medidas se encuentran mejoramientos de pastura, realizar un control efectivo de las desparasitaciones, controlar las montas y no permitir la consanguinidad, que es usual en los sistema de producción de la región en estudio, seleccionar los mejores animales del rebaño y por último, en las épocas criticas garantizar la alimentación del rebaño, es decir, no someter a los animales a estrés alimenticio. Edades al 75 y 95\% de madurez superiores (412 y 918 días) a este estudio fueron reportadas por Simanca et al., (2015).
Respecto a las diferencias halladas $(\mathrm{P} \leq 0.05)$ para el número de crías por parto para Mt7, EM75 y EM95, se encontró que los corderos provenientes de parto únicos alcanzaron un menor peso a la madurez y una mayor tasa de crecimiento que los corderos provenientes de partos duplos, esto puede ser explicado porque a mayor número de crías, menor es el peso al nacimiento y menores son los índices de crecimiento de los corderos, lo que se traduce en diferencias en el peso pre y posdestete (Macedo et al., 2008). Esto puede estar influenciado porque la permanencia en el útero de corderos únicos no tienen competencia por los nutrientes y además tienen mayor espacio (González et al., 2002), lo cual es comprensible puesto que cuando la hembra cría a un solo cordero este tiene mayor disposición de leche y de nutrientes.

Lo anterior nos permite afirmar que cuando el animal proviene de partos únicos, se comporta y crece muchos mejor que aquellos provenientes de partos duplos, es decir, que alcanza un porcentaje de madurez más alto a los siete meses y su edad a la madurez es más corta y por ende se reflejará en un mejor desarrollo corporal del animal.

No se encontró efecto significativo $(P>0.05)$ del número de partos sobre $\beta_{0}, \beta 1$ y $\beta 2$, al igual que para el porcentaje de madurez a los cuatro meses, para la edad al alcanzar el 75 y $95 \%$ de madurez, pero si fue significativo $(\mathrm{P} \leq 0.05)$ para el porcentaje de madurez a los siete meses. Teniendo en cuenta que se encontró que el número de partos de la oveja no afecta el peso asintótico, la tasa de madurez, ni la constante de integración, quiere decir que desde este punto de vista, se pueden tener ovejas con hasta seis partos, que fue el máximo número de partos considerado en el estudio, sin afectar los parámetros anteriores. Lo mismo sucedió para Mt4, EM75, lo que sugiere que la oveja al tener varios partos no genera una variabilidad importante en el sistema de producción para estas variables.

El modelo Brody subestimó el peso al nacimiento de los corderos, esto pudo ser ocasionado por la mucha variabilidad de los pesos al nacer de los animales, quizás por el efecto del tipo de parto.

\section{Conclusiones}

De acuerdo a los valores estimados para porcentaje de madurez y edad a la madurez en la población ovina estudiada, se deben mejorar las condiciones de alimentación, con el fin de que los animales logren un mayor desarrollo corporal en el menor tiempo posible. Los animales nacidos en partos de una sola cría, son más precoces que aquellos nacidos en partos gemela- 
res, por lo tanto alcanzaran su peso adulto en menor tiempo.

\section{Agradecimientos}

Al criadero ovino "Altamar S.A", por facilitar la información para el desarrollo de esta investigación.

\section{Referencias}

Acero-Plazas VM. El bienestar animal en sistemas productivos de ovinos-caprinos en Colombia. Spei Domus. 2014;10(20):57-62. Disponible en: doi: http://dx.doi.org/10.16925/sp.v10i21.918.

Arévalo A, Correa G. Tecnología en la ovinocultura colombiana: estado del arte. Rev Cienc Anim. 2013;6:125-142.

Bahreini MR, Aslaminejad AA, Sharfi AR, Simianer H. Comparison of mathematical models for describing the growth of baluchi sheep. J Agr Sci tech. 2014;14:57-68.

Brody S. Bioenergetics and growth. Amer J of Physical Anthrop. 1946;4(1):116-117.

Costa M, Villarroel A, Sales E, Pinheiro S, Araújo I, Mizubuti I. Curva de crescimento de cordeiros oriundos de três sistemas de produção na Região Nordeste do Brasil. Ciênc Agrár. 2012;33(5):2011-2018. Disponible en: DOI: 10.5433/1679-0359.2012v33n5p2011.

González R, Torres G, Castillo M. Crecimiento de corderos Blackbelly entre el nacimiento y el peso final en el trópico húmedo de México. Vet Méx. 2002; 33(4):443-453.

Hossein-Zadeh NA, Golshani M. Comparison of non-linear models to describe growth of Iranian Guilan sheep. Rev Colomb Cienc Pecu. 2016;29:199-209. Disponible en: doi: 10.17533/udea. rccp.v29n3a05.

Macedo R Arredondo V. Efecto del sexo, tipo de nacimiento y lactancia sobre el crecimiento de ovinos Pelibuey de manejo intensivo. Arch Zootec. 2008;57:219-228.
Malhado CHM Carneiro PLS, Santos PF, Azevedo DMMR, Souza JC, Affonso PRM. Curva de crescimento em ovinos mestiços Santa Inês x Texel criados no Sudoeste do Estado da Bahia. Rev Bras Saúde Prod An. 2008; 9(2):210-218.

Ospina O, Grajales H, Manrique C. Gestión del conocimiento: mayor producción y competitividad. Perspectivas para los sistemas de producción ovino-caprinos. Rev Med Vet. 2011;22:95-113.

Parra RI, Magaña MA, Duarte JH, Téllez G. Caracterización técnica y rentabilidad de granjas ovinas con visión empresarial del departamento del Tolima. Rev Colomb de Cienc Ani. 2014;7(1):64-72.

Santos LM. Crescimento de ovinos de diferentes grupos genéticos sob modelos não lineares convencionais e Iternativos. Dissertação de Mestrado, Universidade Estadual do Sudoeste da Bahia - UESB, Brasil. 2012. Pp 55.

Simanca J, Bustamante M; Medina H, Vergara O. Descripción del crecimiento de ovinos Santa Inés x Criollo manejados en pastoreo extensivo en dos poblaciones de Córdoba, Colombia. Rev Colomb Cienc Pecu 2015; 28(Suplemento:135):

Simanca J, Vergara O, Bustamante M. Descripción del crecimiento de ovinos Santa Inés x Criollo manejados en pastoreo extensivo en dos poblaciones de Córdoba, Colombia. Rev Fac Cs Vets, UCV. 2016;57(1):61-67.

Souza LA, Carneiro PLS, Malhado CHM, Paiva SR, Caire DN, Barreto DLF. Curvas de crescimento em ovinos da raça morada nova criados no estado da Bahia. R Bras Zootec. 2011;40(8):17001705.

Teixeira MR, Cruz JF, Faria HN, Souza ES, Carneiro PL, Malhado CH. Descrição do crescimento de ovinos Santa Inês utilizando modelos não-lineares selecionados por análise multivariada. Rev Bras Saúde Prod Anim. 2016;17(1):26-36.

Ullah M, Amin M, Abbas M. Non-linear regression models to predict the lamb and sheep weight growth. Pak J Nutr. 2013;12(9):865869. 